\title{
JENSEN'S OPERATOR INEQUALITY
}

\author{
Frank Hansen \& Gert K. Pedersen \\ $2^{\text {nd }}$ April, 2002
}

\begin{abstract}
We establish what we consider to be the definitive versions of Jensen's operator inequality and Jensen's trace inequality for real functions defined on an interval. This is accomplished by the introduction of genuine non-commutative convex combinations of operators, as opposed to the contractions considered in earlier versions of the theory, $[\mathbf{9}] \&[\mathbf{3}]$. As a consequence, we no longer need to impose conditions on the interval of definition. We show how this relates to the pinching inequality of Davis [4], and how Jensen's trace inequality generalizes to $C^{*}$-algebras.
\end{abstract}

\section{INTRODUCTION}

If $f$ is a continuous, real function on some interval $I$ in $\mathbb{R}$, we can use spectral theory to define an operator function

$$
f: \mathbb{B}(\mathfrak{H})_{\mathrm{sa}}^{I} \rightarrow \mathbb{B}(\mathfrak{H})_{\mathrm{sa}} \quad \text { where } \quad f(x)=\int f(\lambda) d E_{x}(\lambda)
$$

Here $\mathbb{B}(\mathfrak{H})_{\text {sa }}^{I}$ denotes the convex set of self-adjoint operators on the Hilbert space $\mathfrak{H}$ with spectra in $I$, and $E_{x}$ denotes the spectral measure of $x$. Admittedly it is somewhat dangerous to use the same symbol for the two rather different functions, but the usage is sanctified by time. Whenever necessary we shall try to distinguish between the two by referring either to the function $f$ or to the operator function $f$. As pointed out by $\mathrm{C}$. Davis in [4] a general operator function $F: \mathbb{B}(\mathfrak{H})_{\mathrm{sa}}^{I} \rightarrow \mathbb{B}(\mathfrak{H})$ arises from a spectral function, i.e. $F(x)=f(x)$, if and only if for every unitary $u$

$$
F\left(u^{*} x u\right)=u^{*} F(x) u \quad \text { and } \quad F\left(\begin{array}{ll}
y & 0 \\
0 & z
\end{array}\right)=\left(\begin{array}{cc}
F(y) & 0 \\
0 & F(z)
\end{array}\right)
$$

for every operator $x=y+z$ that decomposes in block form by multiplication by a projection $p$ in its commutant. (N.b. we do not demand that $p$ and $\mathbf{1}-p$ are equivalent.) There is a slight ambiguity in this statement - easily compensated for by its versatility - since by $\mathrm{F}(\mathrm{y})$ we really mean $F$ evaluated at $y$, but now regarded

1991 Mathematics Subject Classification. Primary 46L05; Secondary 46L10, 47A60, 46C15.

Key words and phrases. Operator algebras, operator convex functions, operator inequalities, Jensen inequality.

(C) 2002 by the authors. This paper may be reproduced, in its entirety, for non-commercial purposes. 
as an operator function on $\mathbb{B}(p \mathfrak{H})_{\text {sa }}^{I}$. Put differently, we demand that $p F(y+z)=$ $F(y+z) p$ and that it is independent of $z$. Thus, $p F(y+z)=p F(y+s(\mathbf{1}-p))$ for some, hence any scalar $s$ in $I$. (Davis tacitly assumes that $0 \in I$ and takes $s=0$.)

A continuous function $f: I \rightarrow \mathbb{R}$ is said to be operator convex if

$$
f(\lambda x+(1-\lambda) y) \leq \lambda f(x)+(1-\lambda) f(y)
$$

for each $\lambda$ in $[0,1]$ and every pair of self-adjoint operators $x, y$ on an infinite dimensional Hilbert space $\mathfrak{H}$ with spectra in $I$. The function is said to be matrix convex of order $n$ if the same conditions are satisfied for operators on a Hilbert space of finite dimension $n$. It is well known, cf. [2, Lemma 2.2] that a function is operator convex if and only if it is matrix convex of arbitrary orders.

Just because the function $f$ is convex there is no guarantee that the operator function $f$ is convex. In fact, as shown by Bendat and Sherman in [2], $f$ is operator convex on the interval ] $-1,1$ [ if and only if it has a (unique) representation

$$
f(t)=\beta_{0}+\beta_{1} t+\frac{1}{2} \beta_{2} \int_{-1}^{1} t^{2}(1-\alpha t)^{-1} d \mu(\alpha),
$$

for $\beta_{2} \geq 0$ and some probability measure $\mu$ on $[-1,1]$. In particular, $f$ must be analytic with $f(0)=\beta_{0}, f^{\prime}(0)=\beta_{1}$ and $f^{\prime \prime}(0)=\beta_{2}$. A concise account of this result and its relations to Löwner's theory of operator monotonicity can be found in $[\mathbf{9}]$.

An unexpected phenomenon turns up in relation with convexity in $\mathbb{B}(\mathfrak{H})_{\mathrm{sa}}$. If $\left(a_{1}, \ldots, a_{n}\right)$ is an $n$-tuple of operators with $\sum_{k=1}^{n} a_{k}^{*} a_{k}=\mathbf{1}$, we may think of the element $\sum_{k=1}^{n} a_{k}^{*} x_{k} a_{k}$ as a non-commutative convex combination of the $n$-tuple $\left(x_{1}, \ldots, x_{n}\right)$ in $\mathbb{B}(\mathfrak{H})_{\text {sa }}$. The remarkable fact is that when $f$ is an operator convex function, then the operator function $f$ respects this new structure in the sense that we have the Jensen operator inequality:

$$
f\left(\sum_{k=1}^{n} a_{k}^{*} x_{k} a_{k}\right) \leq \sum_{k=1}^{n} a_{k}^{*} f\left(x_{k}\right) a_{k} .
$$

This result was found in embryonic form by the first author in [6], and used by the two of us to give a review of Löwner's and Bendat-Sherman's theory of operator monotone and operator convex functions in [9]. With hindsight we must admit that we unfortunately proved and used the contractive form $f\left(a^{*} x a\right) \leq a^{*} f(x) a$ for $a^{*} a \leq \mathbf{1}$, this being the seemingly most attractive version at the time. However, this necessitated the further conditions that $0 \in I$ and $f(0) \leq 0$, conditions that have haunted the theory since then. The Jensen inequality for a normal trace on a von Neumann algebra, now for an arbitrary convex function $f$, was found by Brown and Kosaki in [3], still in the contractive version.

It is the aim of the present paper to rectify our omissions and prove the full Jensen inequality, both with and without a trace. This is accomplished by a refinement of previous techniques and by applying some new ideas that also make the presentation more streamlined and easier to follow. 


\section{Main Results}

Theorem 2.1 (Jensen's Operator Inequality).

For a continuous function $f$ defined on an interval $I$ the following conditions are equivalent:

(i) $f$ is operator convex.

(ii) For each natural number $n$ we have the inequality

$$
f\left(\sum_{i=1}^{n} a_{i}^{*} x_{i} a_{i}\right) \leq \sum_{i=1}^{n} a_{i}^{*} f\left(x_{i}\right) a_{i}
$$

for every $n$-tuple $\left(x_{1}, \ldots, x_{n}\right)$ of bounded, self-adjoint operators on an arbitrary Hilbert space $\mathfrak{H}$ with spectra contained in $I$ and every $n$-tuple $\left(a_{1}, \ldots, a_{n}\right)$ of operators on $\mathfrak{H}$ with $\sum_{k=1}^{n} a_{k}^{*} a_{k}=\mathbf{1}$.

(iii) $f\left(v^{*} x v\right) \leq v^{*} f(x) v$ for each isometry $v$ on an infinite-dimensional Hilbert space $\mathfrak{H}$ and every self-adjoint operator $x$ with spectrum in $I$.

(vi) $p f(p x p+s(1-p)) p \leq p f(x) p$ for each projection $p$ on an infinite-dimensional Hilbert space $\mathfrak{H}$, every self-adjoint operator $x$ with spectrum in $I$ and every $s$ in $I$.

Remark 2.2. If the Jensen operator inequality (5) is satisfied for some $n \geq 2$ and for operators on a Hilbert space $\mathfrak{H}$ (of any dimension), then clearly $f$ is operator convex on $\mathbb{B}(\mathfrak{H})$. The point of condition (iii) is that if $\mathfrak{H}$ infinite-dimensional it suffices to take $n=1$. On the other hand it is clear that if (5) is satisfied for some $n$, then (setting $a_{i}=0$ for $i>1$ ) it is also satisfied for $n=1$.

Corollary 2.3 (Contractive Version).

Let $f$ be a continuous function defined on an interval $I$ and suppose that $0 \in I$. Then $f$ is operator convex and $f(0) \leq 0$ if and only if for some, hence every natural number $n$, the inequality (5) is valid for every $n$-tuple $\left(x_{1}, \ldots, x_{n}\right)$ of bounded, self-adjoint operators on a Hilbert space $\mathfrak{H}$ with spectra contained in $I$, and every $n$-tuple $\left(a_{1}, \ldots, a_{n}\right)$ of operators on $\mathfrak{H}$ with $\sum_{k=1}^{n} a_{k}^{*} a_{k} \leq \mathbf{1}$.

Setting $n=1$ we see that $f$ is operator convex on an interval $I$ containing 0 with $f(0) \leq 0$ if and only if

$$
f\left(a^{*} x a\right) \leq a^{*} f(x) a
$$

for every self-adjoint $x$ with spectrum in $I$ and every contraction $a$. This is the original Jensen operator inequality from $[\mathbf{9}]$.

Theorem 2.4 (Jensen's Trace Inequality).

Let $f$ be a continuous function defined on an interval $I$ and let $m$ and $n$ be natural numbers. If $f$ is convex we then have the inequality

$$
\operatorname{Tr}\left(f\left(\sum_{i=1}^{n} a_{i}^{*} x_{i} a_{i}\right)\right) \leq \operatorname{Tr}\left(\sum_{i=1}^{n} a_{i}^{*} f\left(x_{i}\right) a_{i}\right)
$$

for every $n$-tuple $\left(x_{1}, \ldots, x_{n}\right)$ of self-adjoint $m \times m$ matrices with spectra contained in $I$ and every $n$-tuple $\left(a_{1}, \ldots, a_{n}\right)$ of $m \times m$ matrices with $\sum_{k=1}^{n} a_{k}^{*} a_{k}=\mathbf{1}$.

Conversely, if the inequality (7) is satisfied for some $n$ and $m$, where $n>1$, then $f$ is convex. 
Corollary 2.5 (Contractive Version).

Let $f$ be a convex, continuous function defined on an interval $I$, and suppose that $0 \in I$ and $f(0) \leq 0$. Then for all natural numbers $m$ and $n$ we have the inequality (7) for every $n$-tuple $\left(x_{1}, \ldots, x_{n}\right)$ of self-adjoint $m \times m$ matrices with spectra contained in $I$ and every $n$-tuple $\left(a_{1}, \ldots, a_{n}\right)$ of $m \times m$ matrices with $\sum_{k=1}^{n} a_{k}^{*} a_{k} \leq \mathbf{1}$.

Remark 2.6. Let $n=1$ in (7). If $f$ is convex, $0 \in I$ and $f(0) \leq 0$ we have

$$
\operatorname{Tr}\left(f\left(a^{*} x a\right)\right) \leq \operatorname{Tr}\left(a^{*} f(x) a\right)
$$

for every self-adjoint $m \times m$ matrix $x$ with spectrum in $I$ and every $m \times m$ contractive matrix $a$. This is Jensen's trace inequality (for matrices) of Brown and Kosaki [3].

This inequality alone is not sufficient to ensure convexity of $f$, even if $m>1$ (unless $f(0)=0$ is specified in advance). However, for $n>1$ the inequality gives convexity of $f$ as we see from Theorem 2.4. In each case we must assume that $0 \in I$, otherwise the inequality does not make sense. This fact, together with the irrelevant information about $f(0)$, makes the contractive versions of Jensen's inequality less desirable. When we eventually pass to the theory of several variables, cf. [10], the contractive versions mean that 0 belongs to the cube where $f$ is defined, so that part of the coordinate axes must belong to the domain of definition for $f$, and on these we must assume that $f \leq 0$. This assumption is so severe a restraint that it becomes a real problem for the theory.

Theorem 2.7 (Jensen's Trace Inequality for $C^{*}-$ Algebras).

Let $f$ be a convex, continuous function defined on an interval $I$ and let $\mathcal{A}$ be a $C^{*}$-algebra with a finite trace $\tau$. Then the inequality

$$
\tau\left(f\left(\sum_{i=1}^{n} a_{i}^{*} x_{i} a_{i}\right)\right) \leq \tau\left(\sum_{i=1}^{n} a_{i}^{*} f\left(x_{i}\right) a_{i}\right)
$$

is valid for every $n$-tuple $\left(x_{1}, \ldots, x_{n}\right)$ of self-adjoint elements in $\mathcal{A}$ with spectra contained in I and every $n$-tuple $\left(a_{1}, \ldots, a_{n}\right)$ in $\mathcal{A}$ with $\sum_{k=1}^{n} a_{k}^{*} a_{k}=\mathbf{1}$.

If the trace $\tau$ is unbounded, but lower semi-continuous and densely defined, the inequality (9) is still valid if $f \geq 0$, although now some of the numbers may be infinite.

\section{Unital and Unitary Tuples}

Notations. An $n$-tuple $\underline{a}=\left(a_{1}, \ldots, a_{n}\right)$ of operators in $\mathbb{B}(\mathfrak{H})$ is called a contractive column (respectively a unital column) if $\sum_{k=1}^{n} a_{k}^{*} a_{k} \leq \mathbf{1}$ (respectively $\left.\sum_{k=1}^{n} a_{k}^{*} a_{k}=\mathbf{1}\right)$. Contractive rows and unital rows are defined analogously by the conditions $\sum_{k=1}^{n} a_{k} a_{k}^{*} \leq \mathbf{1}$ and $\sum_{k=1}^{n} a_{k} a_{k}^{*}=\mathbf{1}$. We say that $\underline{a}=\left(a_{1}, \ldots, a_{n}\right)$ is a unitary column if there is a unitary $n \times n$ operator matrix $U=\left(u_{i j}\right)$, one of whose columns is $\left(a_{1}, \ldots, a_{n}\right)$. Thus, $u_{i j}=a_{i}$ for some $j$ and all $i$. Unitary rows are defined analogously, cf. [1, Definition 1.1.] Note that an $n$-tuple $\left(a_{1}, \ldots, a_{n}\right)$ is a contractive/unital/unitary row if and only if the adjoint tuple $\left(a_{1}^{*}, \ldots, a_{n}^{*}\right)$ is 
$\mathfrak{H}$ it may happen that an $n$-tuple $\underline{a}$ is a unitary (unital or contractive) column in $\mathbb{B}(\mathfrak{H})$, while $\underline{a}$ is not a unitary (unital or contractive) row in $\mathbb{B}(\mathfrak{H})$. Evidently every unitary column is also a unital column (and similarly every unitary row is a unital row). On the other hand, if $\left(s_{1}, \ldots, s_{n}\right)$ is an $n$-tuple of co-isometries such that $\sum_{k=1}^{n} s_{k}^{*} s_{k}=\mathbf{1}$ (these are the canonical generators for the Cuntz algebra $\mathcal{O}_{n}$ ), then we have a simple example of a unital column that is not unitary. If we insist that a unital column of elements in a unital $C^{*}$-algebra $\mathcal{A}$ should be called a unitary column only if we can choose the unitary in $\mathbb{M}_{n}(\mathcal{A})$, then already for $\mathcal{A}$ commutative, viz. $\mathcal{A}=C\left(\mathbb{S}^{5}\right)$, we have a unital 3 -column that is not a unitary column in $\mathbb{M}_{3}(\mathcal{A})$, cf. [17, Example 14].

Given a unital column $\left(a_{1}, \ldots, a_{n}\right)$ we may regard it as an isometry $\underline{a}: \mathfrak{H} \rightarrow \mathfrak{H}^{n}$, where $\mathfrak{H}^{n}=\oplus_{i=1}^{n} \mathfrak{H}$. Better still we may regard it as a partial isometry $V: \mathfrak{H}^{n} \rightarrow \mathfrak{H}^{n}$, where $V \mid \mathfrak{H}^{n-1}=0$. Evidently the column is unitary precisely when $V$ extends to a unitary operator on $\mathfrak{H}^{n}$, and this happens if and only if the index of $V$ is 0 , in the generalized sense that $\operatorname{dim} \operatorname{ker} V^{*}=(n-1) \operatorname{dim} \mathfrak{H}$. Here $V^{*}\left(\xi_{1}, \ldots, \xi_{n}\right)=$ $a_{1}^{*} \xi_{1}+\cdots+a_{n}^{*} \xi_{n}$ in $\mathfrak{H}$. It follows from [1, Corollary 2.2] that this holds if just one of the operators $a_{i}$ has (generalized) index zero, since in this case $a_{i}=u\left|a_{i}\right|$ for some unitary $u$ on $\mathfrak{H}$. We are then reduced to the situation where one of the operators, say $a_{n}$, is positive, so that with $\underline{b}=\left(a_{1}, \ldots, a_{n-1}\right)$ we can extend $V$ to the unitary operator

$$
U=\left(\begin{array}{cc}
\left(\mathbf{1}-\underline{b}(\underline{b})^{*}\right)^{1 / 2} & \underline{b} \\
-(\underline{b})^{*} & a_{n}
\end{array}\right) .
$$

It follows that every contractive $n$-column can be enlarged to a unitary $(n+1)-$ column simply by setting $a_{n+1}=\left(\mathbf{1}-\sum_{k=1}^{n} a_{k}^{*} a_{k}\right)^{1 / 2}$. In particular, every unital $n$-column can be enlarged to a unitary $(n+1)$-column with $a_{n+1}=0$. As usual we shall refer to this as a unitary dilation of the unital (or contractive) column.

Unitary Dilations. It may sometimes be desirable to know exactly the terms in a unitary dilation of some unital column $\underline{a}=\left(a_{1}, \ldots, a_{n}\right)$. If $n=1$, so that $\underline{a}=a$ for some isometry $a$, the canonical dilation is given by a $2 \times 2$-matrix $U$ having $(a, 0)$ as the second column. For a general unital $n$-column we may regard it as an isometry $\underline{a}: \mathfrak{H} \rightarrow \mathfrak{H}^{n}$, and the unitary dilation $U_{n}$ on $\mathfrak{H} \oplus \mathfrak{H}^{n}$ then has the same form as $U$; in fact

$$
U=\left(\begin{array}{cc}
1-a a^{*} & a \\
-a^{*} & 0
\end{array}\right) \quad \text { and } \quad U_{n}=\left(\begin{array}{cc}
p & \underline{a} \\
-(\underline{a})^{*} & 0
\end{array}\right)
$$

where $p=\mathbf{1}-\underline{a}(\underline{a})^{*}$ is the $n \times n$ projection in $\mathfrak{H}^{n}$ with $p_{i i}=\mathbf{1}-a_{i} a_{i}^{*}$ and $p_{i j}=-a_{i} a_{j}^{*}$ for $i \neq j$. Thus, the canonical dilation of $\left(a_{1}, \ldots, a_{n}\right)$ has the form:

$$
U_{n}=\left(\begin{array}{ccccc}
\mathbf{1}-a_{1} a_{1}^{*} & -a_{1} a_{2}^{*} & \ldots & -a_{1} a_{m}^{*} & a_{1} \\
-a_{2} a_{1}^{*} & \mathbf{1}-a_{2} a_{2}^{*} & \ldots & -a_{2} a_{n}^{*} & a_{2} \\
\vdots & \vdots & & \vdots & \vdots \\
-a_{n} a_{1}^{*} & -a_{n} a_{2}^{*} & \ldots & \mathbf{1}-a_{n} a_{n}^{*} & a_{n} \\
-a_{1}^{*} & -a_{2}^{*} & \ldots & -a_{n}^{*} & 0
\end{array}\right)
$$

As seen from (10), the formula for the canonical dilation of a contractive column is 
Lemma 3.1. Define the unitary matrix $E=\operatorname{diag}\left(\theta, \theta^{2}, \ldots, \theta^{n-1}, 1\right)$ in $\mathbb{M}_{n}(\mathbb{C}) \subset$ $\mathbb{B}\left(\mathfrak{H}^{n}\right)$, where $\theta=\exp (2 \pi \mathrm{i} / n)$. Then for each element $A=\left(a_{i j}\right)$ in $\mathbb{B}\left(\mathfrak{H}^{n}\right)$ we have

$$
\frac{1}{n} \sum_{k=1}^{n} E^{-k} A E^{k}=\operatorname{diag}\left(a_{11}, a_{22}, \ldots, a_{n n}\right) .
$$

Proof. By computation

$$
\left(\frac{1}{n} \sum_{k=1}^{n} E^{-k} A E^{k}\right)_{i j}=\frac{1}{n} \sum_{k=1}^{n}\left(\theta^{j-i}\right)^{k} a_{i j}
$$

and this sum is zero if $i \neq j$, otherwise it is $a_{i i}$.

Corollary 3.2. Let $P$ denote the projection in $\mathbb{M}_{n}(\mathbb{C})$ given by $P_{i j}=n^{-1}$ for all $i$ and $j$, so that $P$ is the projection of rank one on the subspace spanned by the vector $\xi_{1}+\cdots+\xi_{n}$ in $\mathbb{C}^{n}$, where $\xi_{1}, \ldots, \xi_{n}$ are the standard basis vectors. Then with $E$ as in Lemma 3.1 we obtain the pairwise orthogonal projections $P_{k}=E^{-k} P E^{k}$, for $1 \leq k \leq n$, with $\sum_{k=1}^{n} P_{k}=\mathbf{1}$.

Proof. (Cf. [8, Proof of Theorem 2.6]) Evidently each $P_{k}$ is a projection of rank one. Moreover, by Lemma 3.1,

$$
\sum_{k=1}^{n} P_{k}=\sum_{k=1}^{n} E^{-k} P E^{k}=n \operatorname{diag}\left(n^{-1}, \ldots, n^{-1}\right)=\mathbf{1},
$$

from which it also follows that the projections are pairwise orthogonal,

\section{Proofs and Further Results}

Proof of Theorem 2.1. (i) $\Longrightarrow$ (ii) Assume that we are given a unitary $n$-column $\left(a_{1}, \ldots, a_{n}\right)$, and choose a unitary $U_{n}=\left(u_{i j}\right)$ in $\mathbb{B}\left(\mathfrak{H}^{n}\right)$ such that $u_{k n}=a_{k}$. Let $E=\operatorname{diag}\left(\theta, \theta^{2}, \ldots, 1\right)$ as in Lemma 3.1 and put $X=\operatorname{diag}\left(x_{1}, \ldots, x_{n}\right)$, both regarded as elements in $\mathbb{B}\left(\mathfrak{H}^{n}\right)$. Using Lemma 3.1 and the operator convexity of $f$ we then get the desired inequality:

$$
\begin{aligned}
& f\left(\sum_{k=1}^{n} a_{k}^{*} x_{k} a_{k}\right)=f\left(\left(U_{n}^{*} X U_{n}\right)_{n n}\right) \\
= & f\left(\left(\sum_{k=1}^{n} \frac{1}{n} E^{-k} U_{n}^{*} X U_{n} E^{k}\right)_{n n}\right)=\left(f\left(\sum_{k=1}^{n} \frac{1}{n} E^{-k} U_{n}^{*} X U_{n} E^{k}\right)\right)_{n n} \\
\leq & \left(\frac{1}{n} \sum_{k=1}^{n} f\left(E^{-k} U_{n}^{*} X U_{n} E^{k}\right)\right)_{n n}=\left(\frac{1}{n} \sum_{k=1}^{n} E^{-k} U_{n}^{*} f(X) U_{n} E^{k}\right)_{n n} \\
= & \left(U_{n}^{*} f(X) U_{n}\right)_{n n}=\sum a_{k}^{*} f\left(x_{k}\right) a_{k} .
\end{aligned}
$$


Note that for the second equality we use that $f\left(y_{n}\right)=\left(f\left(\operatorname{diag}\left(y_{1}, \ldots, y_{n}\right)\right)\right)_{n n}$ because $f\left(\operatorname{diag}\left(y_{1}, \ldots, y_{n}\right)\right)=\operatorname{diag}\left(f\left(y_{1}\right), \ldots, f\left(y_{n}\right)\right)$.

In the general case where the column is just unital, we enlarge it to the unitary $(n+1)$-column $\left(a_{1}, \ldots, a_{n}, 0\right)$ and choose $x_{n+1}$ arbitrarily, but with spectrum in I. By the first part of the proof we therefore have

$$
\begin{gathered}
f\left(\sum_{k=1}^{n} a_{k}^{*} x_{k} a_{k}\right)=f\left(\sum_{k=1}^{n+1} a_{k}^{*} x_{k} a_{k}\right) \\
\leq \sum_{k=1}^{n+1} a_{k}^{*} f\left(x_{k}\right) a_{k}=\sum_{k=1}^{n} a_{k}^{*} f\left(x_{k}\right) a_{k} .
\end{gathered}
$$

(ii) $\Longrightarrow$ (iii) is trivial.

(iii) $\Longrightarrow$ (iv) Take any self-adjoint operator $x$ with spectrum in $I$ and let $p$ be an infinite-dimensional projection. Then we can find an isometry $v$ (i.e. $v^{*} v=\mathbf{1}$ ) such that $p=v v^{*}$. By assumption $f\left(v^{*} x v\right) \leq v^{*} f(x) v$, whence also

$$
v f\left(v^{*} x v\right) v^{*} \leq v v^{*} f(x) v v^{*}=p f(x) p .
$$

For any monomial $g(t)=t^{m}$ and any $s$ in $I$ we have

$$
\begin{aligned}
p v g\left(v^{*} x v\right) v^{*} p & =p v\left(v^{*} x v\right)^{m} v^{*} p=p\left(v v^{*} x v v^{*}\right)^{m} p \\
\quad=p g(p x p) p & =p g(p x p+s(\mathbf{1}-p)) p .
\end{aligned}
$$

Since $f$ is continuous, it can be approximated by polynomials on compact subsets of $I$, and therefore also $p v f\left(v^{*} x v\right) v^{*} p=p f(p x p+s(\mathbf{1}-p)) p$. Combined with (17) this gives the pinching inequality

$$
p f(p x p+s(\mathbf{1}-p)) p \leq p f(x) p .
$$

If $p$ is a projection of finite rank we can define the infinite dimensional projection $q=p \otimes \mathbf{1}_{\infty}$ on $\mathfrak{H}^{\infty}$. Similarly we let $y=x \otimes \mathbf{1}_{\infty}$ for any given self-adjoint operator $x$ with spectrum in $I$. Since $f(a) \otimes \mathbf{1}_{\infty}=f\left(a \otimes \mathbf{1}_{\infty}\right)$ for any operator $a$ we get by (20) that

$$
\begin{aligned}
p f(p x p+s(\mathbf{1}-p)) p \otimes \mathbf{1}_{\infty} & =q f(q y q+s(\mathbf{1}-q)) q \\
\leq q f(y) q & =p f(x) p \otimes \mathbf{1}_{\infty},
\end{aligned}
$$

which shows that (20) is valid also for projections of finite rank.

(iv) $\Longrightarrow(1)$ Given self-adjoint operators $x$ and $y$ with spectra in $I$ and $\lambda$ in $[0,1]$, define the three elements

$$
X=\left(\begin{array}{ll}
x & 0 \\
0 & y
\end{array}\right), \quad U=\left(\begin{array}{cc}
\lambda^{1 / 2} & (1-\lambda)^{1 / 2} \\
-(1-\lambda)^{1 / 2} & \lambda^{1 / 2}
\end{array}\right), \quad P=\left(\begin{array}{ll}
1 & 0 \\
0 & 0
\end{array}\right)
$$

in $\mathbb{B}\left(\mathfrak{H}^{2}\right)$. Then for some $s$ in $I$ we have by the pinching inequality in (iv) that 
Since

$$
U^{*} X U=\left(\begin{array}{cc}
\lambda x+(1-\lambda) y & \left(\lambda-\lambda^{2}\right)^{1 / 2}(y-x) \\
\left(\lambda-\lambda^{2}\right)^{1 / 2}(y-x) & \lambda y+(1-\lambda) x
\end{array}\right)
$$

it follows that

$$
\begin{aligned}
\left(\begin{array}{cc}
f(\lambda x+(1-\lambda) y) & 0 \\
0 & 0
\end{array}\right) & =P f\left(P U^{*} X U P+s(\mathbf{1}-P)\right) P \\
\leq P U^{*}\left(\begin{array}{cc}
f(x) & 0 \\
0 & f(y)
\end{array}\right) U P & =\left(\begin{array}{cc}
\lambda f(x)+(1-\lambda) f(y) & 0 \\
0 & 0
\end{array}\right) .
\end{aligned}
$$

Proof of Corollary 2.3. If $\sum_{k=1}^{n} a_{k}^{*} a_{k}=b \leq \mathbf{1}$, put $a_{n+1}=(\mathbf{1}-b)^{1 / 2}$. Then we have a unital $(n+1)$-tuple, so with $x_{n+1}=0$ we get

$$
\begin{gathered}
f\left(\sum_{k=1}^{n} a_{k}^{*} x_{k} a_{k}\right)=f\left(\sum_{k=1}^{n+1} a_{k}^{*} x_{k} a_{k}\right) \leq \sum_{k=1}^{n+1} a_{k}^{*} f\left(x_{k}\right) a_{k} \\
=\sum_{k=1}^{n} a_{k}^{*} f\left(x_{k}\right) a_{k}+a_{n+1}^{*} f(0) a_{n+1} \leq \sum_{k=1}^{n} a_{k}^{*} f\left(x_{k}\right) a_{k} .
\end{gathered}
$$

Conversely, if (5) is satisfied for all contractive $n$-tuples, then - a fortiori - it holds for unital $n$-tuples, so $f$ is operator convex; and with $a=x=0$ we see that $f(0) \leq 0 \cdot f(0) \cdot 0=0$.

Proof of Theorem 2.4. Let $x_{k}=\sum_{\mathrm{sp}\left(x_{k}\right)} \lambda E_{k}(\lambda)$ denote the spectral resolution of $x_{k}$ for $1 \leq k \leq n$. Thus, $E_{k}(\lambda)$ is the spectral projection of $x_{k}$ on the eigenspace corresponding to $\lambda$ if $\lambda$ is an eigenvalue for $x_{k}$; otherwise $E_{k}(\lambda)=0$. For each unit vector $\xi$ in $\mathbb{C}^{m}$ define the (atomic) probability measure

$$
\mu_{\xi}(S)=\left(\sum_{k=1}^{n} a_{k}^{*} E_{k}(S) a_{k} \xi \mid \xi\right)=\sum_{k=1}^{n}\left(E_{k}(S) a_{k} \xi \mid a_{k} \xi\right)
$$

for any (Borel) set $S$ in $\mathbb{R}$. Note now that if $y=\sum_{k=1}^{n} a_{k}^{*} x_{k} a_{k}$ then

$$
\begin{aligned}
& (y \xi \mid \xi)=\left(\sum_{k=1}^{n} a_{k}^{*} x_{k} a_{k} \xi \mid \xi\right) \\
= & \left(\sum_{k=1}^{n} \sum_{\mathrm{sp}\left(x_{k}\right)} \lambda E_{k}(\lambda) a_{k} \xi \mid a_{k} \xi\right)=\int \lambda d \mu_{\xi}(\lambda) .
\end{aligned}
$$

If a unit vector $\xi$ is an eigenvector for $y$, then the corresponding eigenvalue is $(y \xi \mid \xi)$, and $\xi$ is also an eigenvector for $f(y)$ with correponding eigenvalue $(f(y) \xi \mid \xi)=$ $f((y \xi \mid \xi))$. In this case we therefore have

$$
\begin{aligned}
& \left(f\left(\sum_{k=1}^{n} a_{k}^{*} x_{k} a_{k}\right) \xi \mid \xi\right)=(f(y) \xi \mid \xi)=f((y \xi \mid \xi)) \\
= & f\left(\int \lambda d \mu_{\xi}(\lambda)\right) \leq \int f(\lambda) d \mu_{\xi}(\lambda) \\
= & \sum^{n}\left(\sum f(\lambda) E_{k}(\lambda) a_{k} \xi \mid a_{k} \xi\right)=\sum^{n}\left(a_{k}^{*} f\left(x_{k}\right) a_{k} \xi \mid \xi\right),
\end{aligned}
$$


where we used (28) and the convexity of $f$ - in form of the usual Jensen inequality - to get the inequality in (29). The result in (7) now follows by summing over an orthonormal basis of eigenvectors for $y$.

Conversely, if (7) holds for some pair of natural numbers $n, m$, where $n>1$, then taking $a_{i}=0$ for $i \geq 2$ we see that the inequality holds for $n=2$. Given $s, t$ in $I$ and $\lambda$ in $[0,1]$ we define $x=s \mathbf{1}_{m}$ and $y=t \mathbf{1}_{m}$ in $\mathbb{M}_{m}(\mathbb{C})$. Then with $a=\lambda^{1 / 2} \mathbf{1}_{m}$ and $b=(1-\lambda)^{1 / 2} \mathbf{1}_{m}$ we get by $(7)$ that

$$
\begin{aligned}
& m f(\lambda x+(1-\lambda) t)=\operatorname{Tr}\left(f(\lambda x+(1-\lambda) t) \mathbf{1}_{m}\right) \\
= & \operatorname{Tr}\left(f\left(a^{*} x a+b^{*} y b\right)\right) \leq \operatorname{Tr}\left(a^{*} f(x) a+b^{*} f(y) b\right) \\
= & \operatorname{Tr}\left((\lambda f(s)+(1-\lambda) f(t)) \mathbf{1}_{m}\right)=m(\lambda f(s)+(1-\lambda) f(t)),
\end{aligned}
$$

which shows that $f$ is convex.

Continuous Fields of Operators. Let $\mathcal{A}$ be a $C^{*}$-algebra of operators on some Hilbert space $\mathfrak{H}$ and $T$ a locally compact Hausdorff space. We say that a family $\left(a_{t}\right)_{t \in T}$ of operators in the multiplier algebra $M(\mathcal{A})$ of $\mathcal{A}$, i.e. the $C^{*}$-algebra $\{a \in \mathbb{B}(\mathfrak{H}) \mid \forall x \in \mathcal{A}: x a+a x \in \mathcal{A}\}$, is a continuous field, if the function $t \rightarrow a_{t}$ is norm continuous. If $\mu$ is a Radon measure on $T$ and the function $t \rightarrow\left\|a_{t}\right\|$ is integrable, we can then form the Bochner integral $\int_{T} a_{t} d \mu(t)$, which is the unique element in $M(\mathcal{A})$ such that

$$
\varphi\left(\int_{T} a_{t} d \mu(t)\right)=\int_{T} \varphi\left(a_{t}\right) d \mu(t) \quad \varphi \in \mathcal{A}^{*} .
$$

If all the $a_{t}$ 's belong to $\mathcal{A}$ then also $\int_{T} a_{t} d \mu(t)$ belongs to $\mathcal{A}$. If $\left(a_{t}^{*} a_{t}\right)_{t \in T}$ is integrable with integral 1 we say that $\left(a_{t}\right)_{t \in T}$ is a unital column field.

The transition from sums to continuous fields is prompted by the nature of the proof of Theorem 4.1, but the interested reader can easily verify that also Theorem 2.1 is valid for continuous fields. We finally note that the restriction to continuous fields is handy, but not necessary. In [10] we shall generalize the setting to arbitrary weak* measurable fields.

Centralizers. Recall that the centralizer of a positive functional $\varphi$ on a $C^{*}$-algebra $\mathcal{A}$ is the closed ${ }^{*}$-subspace $\mathcal{A}^{\varphi}=\{y \in \mathcal{A} \mid \forall x \in \mathcal{A}: \varphi(x y)=\varphi(y x)\}$. In general this is not an algebra, but if $y_{1}, \ldots, y_{n}$ are pairwise commuting, self-adjoint elements in $\mathcal{A}^{\varphi}$ then the $C^{*}$-algebra they generate is contained in $\mathcal{A}^{\varphi}$. Evidently the size of $\mathcal{A}^{\varphi}$ measures the extent to which $\varphi$ is a trace. The fact we shall utilize is that even if an element $x$ is outside $\mathcal{A}^{\varphi}$ the functional will behave "trace-like" on the subspace spanned by $\mathcal{A}^{\varphi} x \mathcal{A}^{\varphi}$.

If $\varphi$ is unbounded, but lower semi-continuous on $\mathcal{A}_{+}$and finite on the minimal dense ideal $K(\mathcal{A})$ of $\mathcal{A}$, we define $\mathcal{A}^{\varphi}=\{y \in \mathcal{A} \mid \forall x \in K(\mathcal{A}): \varphi(x y)=\varphi(y x)\}$.

Theorem 4.1. Let $\left(x_{t}\right)_{t \in T}$ be a bounded, continuous field on a locally compact Hausdorff space $T$ consisting of self-adjoint elements in a $C^{*}$-algebra $\mathcal{A}$ with $\operatorname{sp}\left(x_{t}\right) \subset I$. Furthermore, let $\left(a_{t}\right)_{t \in T}$ be a unital column field in $M(\mathcal{A})$ with respect to some Radon measure $\mu$ on $T$. Then for each continuous, convex func- 
$y=\int_{T} a_{t}^{*} x_{t} a_{t} d \mu(t)$ in its centralizer $\mathcal{A}^{\varphi}$, i.e. $\varphi(x y)=\varphi(y x)$ for all $x$ in $\mathcal{A}$, we have the inequality:

$$
\varphi\left(f\left(\int_{T} a_{t}^{*} x_{t} a_{t} d \mu(t)\right)\right) \leq \varphi\left(\int_{T} a_{t}^{*} f\left(x_{t}\right) a_{t} d \mu(t)\right) .
$$

If $\varphi$ is unbounded, but lower semi-continuous on $\mathcal{A}_{+}$and finite on the minimal dense ideal $K(\mathcal{A})$ of $\mathcal{A}$, the result still holds if $f \geq 0$, even though the function may now attain infinite values.

Proof. Let $\mathcal{C}=C_{o}(S)$ denote the commutative $C^{*}$-subalgebra of $\mathcal{A}$ generated by $y$, and let $\mu_{\varphi}$ be the finite Radon measure on the locally compact Hausdorff space $S$ defined, via the Riesz representation theorem, by

$$
\int_{S} z(s) d \mu_{\varphi}(s)=\varphi(z) \quad z \in \mathcal{C}=C_{o}(S) .
$$

Since for all $(x, z)$ in $M(\mathcal{A})_{+} \times \mathcal{C}_{+}$we have $\varphi(x z)=\varphi\left(z^{1 / 2} x z^{1 / 2}\right)$ it follows that

$$
0 \leq \varphi(x z) \leq\|x\| \varphi(z)
$$

Consequently the functional $z \rightarrow \varphi(x z)$ on $\mathcal{C}$ defines a Radon measure on $S$ dominated by a multible of $\mu_{\varphi}$, hence determined by a unique element $\Phi(x)$ in $L_{\mu_{\varphi}}^{\infty}(S)$. By linearization this defines a conditional expectation $\varphi: M(\mathcal{A}) \rightarrow L_{\mu_{\varphi}}^{\infty}(S)$ (i.e. a positive, unital module map) such that

$$
\int_{S} z(s) \Phi(x)(s) d \mu_{\varphi}(s)=\varphi(z x), \quad z \in \mathcal{C} \quad x \in M(\mathcal{A})
$$

Inherent in this formulation is the fact that if $z \in \mathcal{C}=C_{o}(S)$, then $\Phi(z)$ is the natural image of $z$ in $L_{\mu_{\varphi}}^{\infty}(S)$. In particular, $z(s)=\Phi(z)(s)$ for almost all $s$ in $S$.

Observe now that since the $C^{*}$-algebra $C_{o}(I)$ is separable, we can for almost every $s$ in $S$ define a Radon measure $\mu_{s}$ on $I$ by

$$
\int_{I} g(\lambda) d \mu_{s}(\lambda)=\Phi\left(\int_{T} a_{t}^{*} g\left(x_{t}\right) a_{t} d \mu(t)\right)(s) \quad g \in C(I) .
$$

As $\int_{T} a_{t}^{*} a_{t} d \mu(t)=\mathbf{1}$, this is actually a probability measure. If we take $g(\lambda)=\lambda$, then

$$
\int_{I} \lambda d \mu_{s}(\lambda)=\Phi\left(\int_{T} a_{t}^{*} x_{t} a_{t} d \mu(t)\right)(s)=\Phi(y)(s)=y(s) .
$$

Since $y \in C$ we get by (37) and (36) - using also the convexity of $f$ in form of the standard Jensen inequality - that

$$
\begin{aligned}
& f(y)(s)=f(y(s))=f\left(\int_{I} \lambda d \mu_{s}(\lambda)\right) \\
& \leq \quad \int f(\lambda) d \mu_{s}(\lambda)=\Phi\left(\int a_{t}^{*} f\left(x_{t}\right) a_{t} d \mu(t)\right)(s) .
\end{aligned}
$$


Integrating over $s$, using (35), now gives the desired result:

$$
\begin{aligned}
\varphi(f(y)) & =\int_{S} f(y)(s) d \mu_{\varphi}(s) \\
& \leq \int_{S} \Phi\left(\int_{T} a_{t}^{*} f\left(x_{t}\right) a_{t} d \mu(t)\right)(s) d \mu_{\varphi}(s) \\
& =\int_{T} \int_{S} \Phi\left(a_{t}^{*} f\left(x_{t}\right) a_{t}\right)(s) d \mu_{\varphi}(s) d \mu(t) \\
& =\int_{T} \varphi\left(a_{t}^{*} f\left(x_{t}\right) a_{t}\right) d \mu(t)=\varphi\left(\int_{T} a_{t}^{*} f\left(x_{t}\right) a_{t} d \mu(t)\right) .
\end{aligned}
$$

Having proved the finite case, let us now assume that $\varphi$ is unbounded, but lower semi-continuous on $\mathcal{A}_{+}$and finite on the minimal dense ideal $K(\mathcal{A})$ of $\mathcal{A}$. This by definition - means that $\varphi(x)<\infty$ if $x \in \mathcal{A}_{+}$and $x=x e$ for some $e$ in $\mathcal{A}_{+}$, because $K(\mathcal{A})$ is the hereditary ${ }^{*}$-subalgebra of $\mathcal{A}$ generated by such elements, cf. $[\mathbf{1 7}, 5.6 .1]$. Restricting $\varphi$ to $\mathcal{C}$ we therefore obtain a unique Radon measure $\mu_{\varphi}$ on $S$ such that

$$
\int_{S} z(t) d \mu_{\varphi}(t)=\varphi(z) \quad y \in \mathcal{C} .
$$

Inspection of the proof above now shows that the Jensen trace inequality still holds if only $f \geq 0$, even though $\infty$ may now occur in the inequality.

Proof of Theorem 2.7. Evidently this (like Theorem 2.4) is a special case of Theorem 4.1, where the continuous field is replaced by a finite sum and the functional $\varphi$ is a trace, so that $\mathcal{A}^{\varphi}=\mathcal{A}$. 


\section{REFERENCES}

[1] Huzihiro Araki \& Frank Hansen, Jensen's operator inequality for functions of several variables, Proceedings of the American Mathematical Society 128 (2000), 2075-2084.

[2] Julius Bendat \& Seymour Sherman, Monotone and convex operator functions, Transactions of the American Mathematical Society 79 (1955), 58-71.

[3] Lawrence G. Brown \& Hideki Kosaki, Jensen's inequality in semi-finite von Neumann algebras, Journal of Operator Theory 23 (1990), 3-19.

[4] Chandler Davis, A Schwarz inequality for convex operator functions, Proceedings of the American Mathematical Society 8 (1957), 42-44.

[5] Chandler Davis, Notions generalizing convexity for functions on spaces of matrices, "Proceedings of Symposia in Pure Mathematics" 7, American Mathematical Society, 1963, pp. 187201.

[6] Frank Hansen, An operator inequality, Mathematische Annalen 246 (1980), 249-250.

[7] Frank Hansen, Operator inequalities associated with Jensen's inequality, "Survey on Classical Inequalities", editor T.M. Rasselas, Kluwer Academic Publishers, 2000, pp. 67-98.

[8] Frank Hansen, Operator monotone functions of several variables, Mathematical Inequalities \& Applications (to appear).

[9] Frank Hansen \& Gert K. Pedersen, Jensen's inequality for operators and Löwner's theorem, Mathematische Annalen 258 (1982), 229-241.

[10] Frank Hansen \& Gert K. Pedersen, Jensen's trace inequality in several variables, Preprint.

[11] Erhard Heinz, Beiträge zur Störungstheorie der Spektralzerlegung, Mathematische Annalen 123 (1951), 415-438.

[12] Richard V. Kadison \& John R. Ringrose, "Fundamentals of the Theory of Operator Algebras", vol I-II, Academic Press, San Diego, 1986 (Reprinted by AMS in 1997).

[13] Fritz Kraus, Über konvexe Matrixfunktionen, Mathematische Zeitschrift 41 (1936), 18-42.

[14] Karl Löwner, Über monotone Matrixfunktionen, Mathematische Zeitschrift 38 (1934), 177216.

[15] Elliott H. Lieb \& Gert K. Pedersen, Multivariable convex trace functions, Reviews in Mathematical Physics, to appear.

[16] Masanori Ohya \& Dénes Petz, "Quantum Entropy and its Use", Texts and Monographs in Physics, Springer Verlag, Heidelberg, 1993.

[17] Gert K. Pedersen, "C $C^{*}-$ Algebras and their Automorphism Groups", LMS Monographs 14, Academic Press, San Diego, 1979.

[18] Gert K. Pedersen, Extreme $n$-tuples of elements in $C^{*}$-algebras, Bulletin of the London Mathematical Society 19 (1987), 264-270.

[19] Dénes Petz, Spectral scale of self-adjoint operators and trace inequalities, Journal of Mathematical Analysis and Applications 109 (1985), 74-82.

[20] Shôichirô Sakai, " $C^{*}-$ Algebras and $W^{*}-$ Algebras", Springer Verlag, Heidelberg, 1971, reprinted 1997.

Department of Economics, University of Copenhagen, Studiestrede 6, DK-1455

Copenhagen K, Denmark \& Department of Mathematics, University of Copenhagen, Universitetsparken 5, DK-2100 Copenhagen $\varnothing$, Denmark

E-mail address: frank.hansen@ econ.ku.dk \& gkped@ math.ku.dk 